\title{
Introduction to Traffic Management for Broadband ISDN
}

\section{Yoon Chang \\ David Su \\ Shukri Wakid}

U.S. DEPARTMENT OF COMMERCE Technology Administration National Institute of Standards and Technology

Gaithersburg, MD 20899 



\section{Introduction to Traffic Management for Broadband ISDN}

\section{Yoon Chang \\ David Su \\ Shukri Wakid}

U.S. DEPARTMENT OF COMMERCE Technology Administration

National Institute of Standards

and Technology

Gaithersburg, MD 20899

December 1993

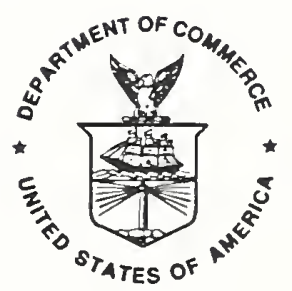

U.S. DEPARTMENT OF COMMERCE Ronald H. Brown, Secretary

TECHNOLOGY ADMINISTRATION

Mary $L$ Good, Under Secretary for Technology

NATIONAL INSTITUTE OF STANDARDS

AND TECHNOLOGY

Arats Prabhakar, Director 


\title{
INTRODUCTION TO TRAFFIC MANAGEMENT FOR BROADBAND ISDN
}

Yoon Chang, David Su, Shukri Wakid

\begin{abstract}
This paper presents an overview of issues related to the traffic management for the Broadband Integrated Services Digital Networks (B-ISDN). B-ISDN is an emerging high bandwidth telecommunications infrastructure with transmission speeds in the range of mega-bit to giga-bits per second. This paper first analyzes traffic profiles in a B-ISDN environment and their impact on traffic management. It then presents an overview of traffic management for existing communications protocols, and discusses, in depth, how it can be applied to the B-ISDN environment. Finally, the paper gives a brief review of the current work on B-ISDN traffic management by the National Accredited Standards Committee $\mathrm{T} 1$ and the International Telegraph and Telephone Consulative Committee (CCITT).
\end{abstract}




\section{INTRODUCTION TO TRAFFIC MANAGEMENT FOR BROADBAND ISDN}

Table of Contents

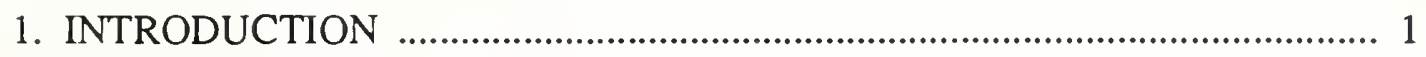

2. B-ISDN ENVIRONMENT AND ATM NETWORKS...................................... 2

3. OVERVIEW OF TRAFFIC MANAGEMENT …......................................... 3

3.1. Flow Control ...................................................................................... 3

3.2 Congestion Control .................................................................................. 4

3.3 Traffic Control ...................................................................................... 5

4. TRAFFIC MANAGEMENT FOR B-ISDN PROTOCOLS ............................. 5

4.1 B-ISDN Reference Configuration and Protocol Standards .................... 5

4.2 Possible Traffic Management Mechanisms for B-ISDN Protocols ...... 7

4.2.1. Flow Control ........................................................................... 7

4.2.2. Congestion Control............................................................. 8
a). Cell Discard
b). Forward Congestion Notification (FCN)
c). Backward Congestion Notification (BCN)

4.2.3. Media Access Control ........................................................... 9

4.2.4. Traffic Control .................................................................... 9

a). Connection Admission Control (CAC)

b). Traffic Policing Function

c) Traffic Shaping Control

4.3 Fairness of Traffic Management Mechanisms

5. CURRENT TRAFFIC MANAGEMENT PROPOSALS FOR B-ISDN

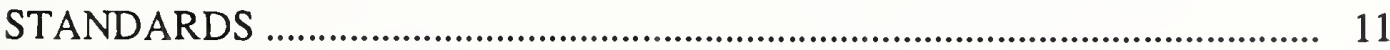

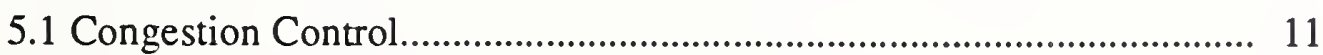

5.2 Traffic Control ................................................................................ 12

5.3 Media Access Control (Generic Flow Control) ....................................... 13

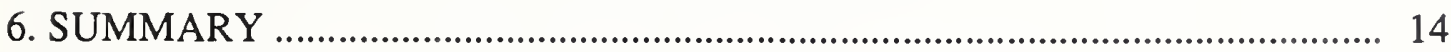

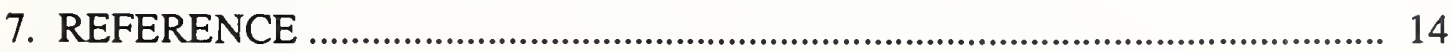





\title{
INTRODUCTION TO TRAFFIC MANAGEMENT FOR BROADBAND ISDN
}

\author{
Yoon Chang, David Su, Shukri Wakid
}

\section{INTRODUCTION}

Advances in computer and communications technology have increased the need for high performance communications networks. The U.S. Government High Performance Computing and Communication initiative (HPCC) will speed up the development of new applications that will require transparent access to large amounts of information across wide geographical areas Examples.of such information include high resolution imaging and graphics displays with integrated data, video and audio.

The ANSI Accredited Standards Committee T1 for Telecommunications in the U. S. and the International Telegraph and Telephone Consultative Committee (CCITT) in the international community are currently developing standards for Broadband Integrated Services Digital Network (B-ISDN) for data rates up to $622 \mathrm{mega}-$ bits/second. The basic architecture for B-ISDN is based on the Asynchronous Transfer Mode (ATM)[S1,S3,S7] and Synchronous Optical Network (SONET) standards [S8]. SONET is a physical layer interface and digital transmission protocol specification for use with high speed optical fibers. ATM is part of a data link layer packet switching technique for B-ISDN services. ATM allows for sharing of network resources by mutiplexing fixed size packet, called "cells", from multiple sources over the same physical transmission facility. This approach allows greater network resource utilization than circuit switch techniques.

An important ATM architectural issue, traffic management, remains to be addressed by the standards groups.

Similar issues have been studied and schemes implemented in traditional data networks for some time. These schemes may not be directly applicable in an ATM environment due to unique characteristics of the B-ISDN such as very high speed switching and transmission, wide range of applications with varying traffic characteristics and service requirements. New ideas and approaches in traffic management must be developed to satisfy these requirements.

This paper represents the results of ongoing research at NIST on B-ISDN protocols. We first analyze the traffic profiles in a B-ISDN environment and their impact on traffic management. A traffic management survey for existing communications protocols is then followed with an in-depth discussion of how such management can be applied to the B-ISDN environment. Finally, a brief review of current work on B-ISDN traffic management by the standards groups 
in ASC T1 and CCITT is presented. We also provide an extensive list of references as an overview of work being done in the subject area.

\section{B-ISDN ENVIRONMENT AND ATM NETWORKS}

There are several unique characteristics which distinguish ATM networks from other high speed networks under study: simple protocol, high resource sharing, and wide range of services. The ATM protocol is a lightweight protocol with no error recovery and simple flow control mechanisms. The use of fixed size cell allows high speed switching, and makes sharing of resources easy. An ATM network can support connections at any bit rate up to the capacity of the physical interface, thus make "bandwidth on demand" possible. ATM is expected to support a wide range of traffic types from voice and data to image and high definition video signals. A typical connection may involve multi-media data including a mixture of aforementioned traffic types. For the purpose of traffic management, the traffic profiles of these applications can be classified in terms of the following parameters:

- Bit rate - Is the bit rate of the traffic source constant (CBR) or variable (VBR)?

- Timing information - Does traffic carry timing information? Shall the timing relation between source and destination be preserved?

- Data integrity - Is all information essential, or is some cell loss tolerable?

Example applications of having CBR traffic include circuit emulation, voice, and constant bit rate video. They all have a strong timing requirement, but with varying degree of sensitivity to data integrity. Loss of cells transmitted may degrade the quality of voice or video, but is not fatal to application. Loss of cells for circuit emulation, however, may result in loss of synchronization.

Packetized video is an example application having VBR traffic with moderate timing requirements. Its requirement for data integrity depends on the acceptable level of video quality.

Synchronous data applications, such as a connection between two Local Area Networks or two Wide Area Networks, is an example of an application having VBR traffic with no timing requirements. Data integrity is essential unless the application can handle error recovery on an end-to-end basis.

Since B-ISDN needs to support such a wide variety of services, the demand on network resources will vary over time. While ATM cell switching is an effective technique for statistical resource sharing under varying traffic volume, it is essential that proper traffic management be employed to limit the probability and duration of traffic overloads on shared resources.

When one studies statistics over a set of time spans, the traffic patterns observed will be different 
for data over a long period, for example, monthly data, than those on a second by second bases. Since an ATM network is expected to carry mixed types of traffic, there must be several traffic management schemes for various time scales, each addressing a unique aspects of network traffics.

- Network Provisioning Level: The traffic measurement of interest here is the aggregate network traffic in relation to the network capacity. One way to reduce the need for other microlevel traffic management is to over engineer the network with excess capacity. Network capacity planning and resource deployment processes provide long term traffic management in time scales of months to years.

- Call Level: The ATM network may accept or deny a call based on dynamic network load. This form of traffic management is applicable in time scales of minutes to hours.

- Dialog Level: A connection may invoke a sequence of transactions, for example, starting with voice and video real time data, followed by file transfers.. Each of these activities is in the seconds to minutes time scale. An example of traffic management is a fast resource reservation mechanism that allow the network to dynamically adjust the resources allocated to a particular connection.

- Burst Level: User activities at the time scale of milliseconds to seconds in which cells come in bursts, for example, cells for a file segment or a video frame.

- Cell Level: Traffic is measured in terms of instantaneous peak rate. Traffic control would be on individual cell basis, such as buffering and dropping of cells.

In summary, traffic management for the ATM networks must consider various types of services as well as traffic variations over time. The high data rate, the variations in traffic type and in requirements for data integrity and latency, present a great challenge in the development of standards.

\section{OVERVIEW OF TRAFFIC MANAGEMENT}

We view traffic management as consisting of three interrelated aspects: flow control, congestion control, and traffic control. Although these terms are very often mentioned as having the same meanings, in general, a congestion control implies reactive actions taken to minimize the intensity, spread, and duration of congestion when a network encounters a traffic overload condition; traffic control and flow control are preventive actions taken to avoid congestion by monitoring and smoothing traffic. These controls are generally applied at the data link, network, and transport layers of the OSI protocol stack.

\subsection{Flow Control}

Flow control is a technique to ensure that a data transmitting entity does not overload the 
network, the receiving entity, or both. Flow control is the method whereby a network or the receiving entity can exercise a typical back-pressure control or other necessary actions on a data source, in order that the data flows through the network at a controlled rate.

Traditional flow control techniques were designed for and used in low speed packet switched data networks (PDNs) that carry mostly non-real-time data. Typical flow control schemes include stop-and-go and sliding window. They may be employed by a network on a node-by-node basis, such as X.25 PDNs, or on an end-to-end basis, such as Internet TCP and OSI Transport protocols. In the following discussion, the term "control node" represents the entity that exercises the control. This may be a transit network node or the final destination.

The stop-and-go scheme is the simplest form of flow control. The control node indicates its willingness to accept another frame by sending an acknowledgment of a frame received from a source node. The source must wait until it receives an acknowledgment of the previous frame from a control node. By way of doing this, the control node can stop the flow of data traffic by simply withholding acknowledgment. Thus, the send, stop, wait and go steps can repeatedly cycle between a source and a control node.

In sliding window schemes, a source node sends multiple frames, called a window of frames, within the limit of buffer sizes allocated in the control node. Both source and control nodes keep track of the number of frames outstanding in the network for each virtual circuit using some acknowledgment mechanisms. A control node controls the number of frames outstanding by varying the rate of acknowledgment. Instead of doing the frame acknowledgment, some networks use a "credit" mechanism. The destination would give the sender a credit of how many frames or bytes it could send. When the sender exhausted the credit, it would stop and wait for new credit authorization from the destination.

\subsection{Congestion Control}

Congestion control is a mechanism to deal with the situations in which the total offered traffic approaches or exceeds the network capacity. Congestion can occur when several sources simultaneously send bursts of data using up the resources of a network node. The congestion may be due to limits in the buffer pools, the capacity of the trunk links, or the switching capacity of the intervening network nodes.. Congestion control mechanism in a network operates in the following manner: The packets are transferred if the required bandwidth is smaller than the bandwidth currently available. Otherwise, the packets are stored in the buffer. If the buffer is full, the incoming packets are dropped. Congestion notices may be sent forward to the destination or backward to the source. The former is called Forward Congestion Notification, and the later called Backward Congestion Notification.

Recent trend in congestion control for high speed network is to discard all excess data. However, discarding a cell which is part of a large message may cause the entire message to be 
retransmitted, thus generating additional traffic on the already overloaded network. A more intelligent method involving coordination with the source node is necessary.

Congestion control must work with other congestion avoidance measures as described in the sections on flow control and traffic control.

\subsection{Traffic Control}

In addition to the flow control and congestion control mechanisms described above, there are other traffic management methods that can be used in conjunction with them. We use the term "Traffic Control" for this class of controls. It includes preventive actions to avoid congestion and reactive actions as congestion occur. Example of these actions are: 1) allocating resources in advance (for example, bandwidth allocation, buffer allocation), 2) classifying messages into priority classes to allow selective discards, 3 ) controlling the rate of data entering the network to reduce the burstiness of traffic, and 4) limiting entrance of new users.

\section{TRAFFIC MANAGEMENT FOR B-ISDN PROTOCOLS}

How the traffic management schemes described above could be used in a high speed and high bandwidth network environment are the issues to be addressed in B-ISDN standards. There are three "layers" of protocols being developed for B-ISDN: the ATM Adaptation layer (AAL), the ATM layer, and the physical layer. The AAL protocol applies end-to-end between two applications of B-TEs, and the ATM applies hop-to-hop between any two ATM nodes. This section discusses traffic management issued on these protocols.

Section 4.1 gives a brief overview of the protocols under consideration. Section 4.2 discusses possible mechanisms could be used by these protocols. The discussion focuses in the three areas of traffic management discussed in Section 3: flow control, congestion control, and traffic control.

\subsection{B-ISDN Reference Configuration and Protocol Standards}

CCITT has defined a set of abstract reference points for a model of the B-ISDN network, to define precisely the points where standards are applicable. Figure 1 depicts the model presented in the CCITT Draft Recommendation I.371 [S4] for traffic management. There are two major spheres of control: the Customer premises and the Network. The interface between a customer premises and a network is referred to as the User-Network Interface (UNI), and the interface between networks is called the Network-Network Interface (NNI). The UNI is represented by the reference points $S_{B}$ and $T_{B}$, as well as the link between Customer Promises equipment and 
the Network which has no designated reference point name. The Customer Premises equipment includes Network Termination (B-NT1 and B-NT2) and Broadband Terminal Equipment (B-TE). The smaller circle in the customer premise enclosing B-NT2 and B-TEs may contain one or more pieces of Terminal Equipment connected to B-NT2 using a star, bus, or ring configuration. If a shared medium such as ring or bus is used, the configuration also requires an access control mechanism to manage the traffic within the premise, as well as the traffic from/to the network.

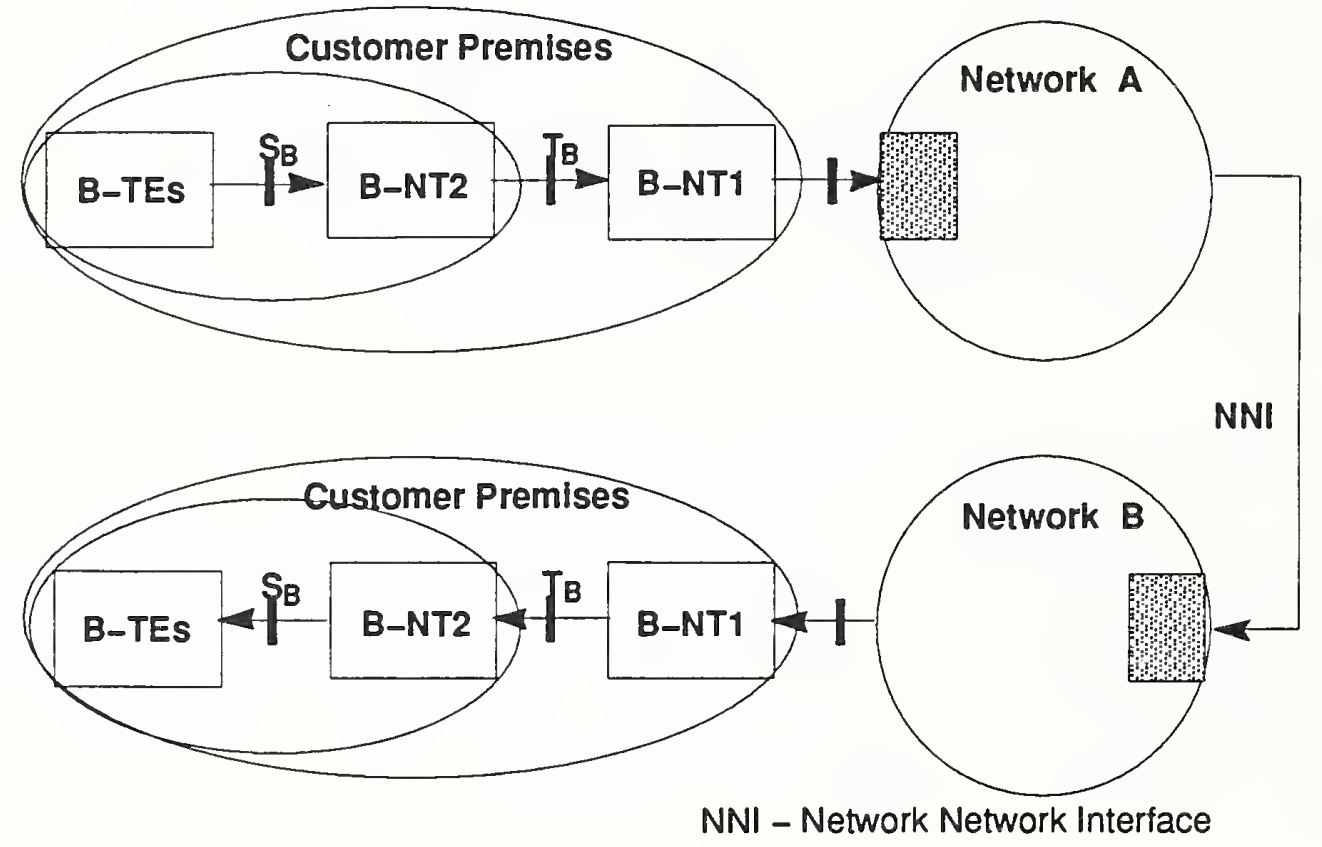

Figure 1. B-ISDN Reference Configuration

The proposed protocols for the UNI interface are shown in Figure 2. The Synchronous Optical Network (SONET) protocol at the Physical "Layer" (PHY) handles the transport of data over a physical medium, such as optical fibers. The ATM "Layer" provides for the transparent and sequential transfer of fixed-size data units called cells. The AAL "Layer" protocols handle the segmentation of messages into cells and reassembly of cells; it also provides whatever actions are required to implement the various type of services it supports. The format of ATM cells is shown in Figure $2 \mathrm{~b}$ and $2 \mathrm{c}$. Note that the fields of interest to traffic management at the ATM level are the Generic Flow Control (GFC), Payload Type, and Cell Loss Priority. 


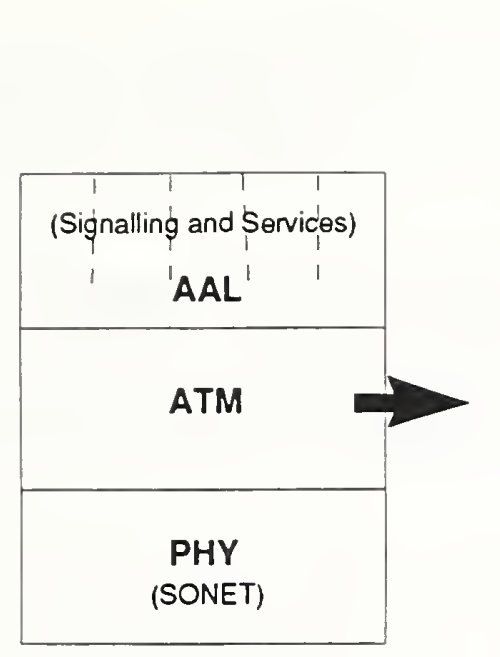

(a) Protocol Stack

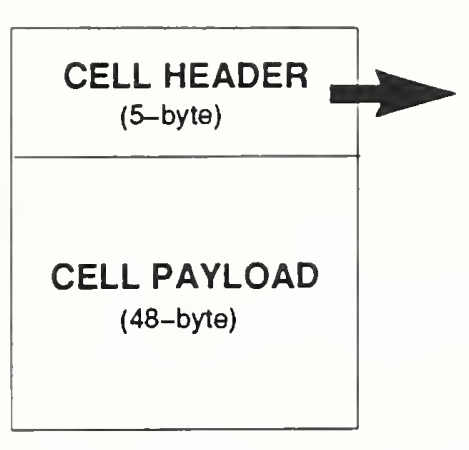

(b) ATM Cells

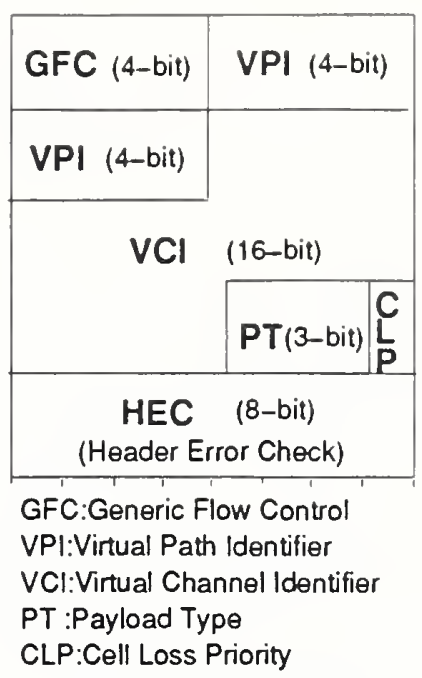

(c) ATM Cell Header

Figure 2. B-ISDN Protocol Stack and ATM Cell Structure

\subsection{Possible Traffic Management Mechanisms for B-ISDN Protocols}

This section discusses the applicability of traffic management mechanisms to B-ISDN protocols and the standard reference points at which they are applicable General requirements for each type of control are also described.

\subsubsection{Flow Control}

Flow control using the node-to-node window rotation (or sliding window) mechanism will not be very effective at the anticipated B-ISDN network transmission rate. The window size, or the amount of data in transit, would have to be very large. This would require high network buffering resource, and reduce the effect of closing a "window." For example, for a 100 mega-bits/second connection with a round trip propagation delay of 5 milliseconds (for a distance of $500 \mathrm{~km}$ ), a window size greater then 60,000 bytes would be required to effectively utilize the available bandwidth. As a result, there is no plan to use window rotation at the ATM cell level. However, some type of end-to-end flow control between the two B-TEs is necessary to respond to network's congestion notification in the ATM cells. This control would have to be done at the AAL level or higher for which a B-ISDN network has no direct control. Due to the propagation and processing delays, the effectiveness of higher layer end-to-end control needs further study.

It would be feasible, however, to perform a stop-and-go backward flow control, in particular at the $T_{B}$ reference point and NNI. This mechanism is discussed further in Section 4.2.2.c on Backward Congestion Control. 


\subsubsection{Congestion Control}

Congestion Control is to be used for immediate relief when congestion has been detected. Therefore the control must have fast reaction time for the mechanism to be effective. Dynamic routing to avoid congested path on a cell by cell basis is not feasible, as the ATM cell does not carry cell sequence number to handle cells arrive out of order. There are three possible ways to exercise the control:

a) Cell Discard: A congested node could selectively discard ATM cells. The main issue is which cells to discard? The general criteria should be the maintenance of the subscribed Quality of Service and fairness to all users of the same service class. For example, customers subscribing to variable bit rate service with provisions for cell discard could be selected first. The application at the source would mark non-critical cells as candidates for discard in the Cell Loss Priority field in the ATM header. Cells from those connections that exceed the negotiated service rate could also be marked for discard. Of cause this assumes the application has a way of telling the AAL which part of data are dispensable.

b) Forward Congestion Notification (FCN): FCN is used to notify the destination B-TE that the cell passed through a congested path and that the destination needs to exercise flow control. The notification mechanism could be done by setting a flag in either the ATM header (in the GFC or PT fields), or inserting a special cell for network Operations, Administrations, and Maintenance $(\mathrm{OA} \& \mathrm{M})$. In addition, protocol primitives for interlayer interface must be able to convey the congestion status from the ATM layer up to higher layers, including the AAL.

This type of control requires cooperation from the user, since it is up to the destination node to take action upon receipt of the FCN. A network could offer a multigrade service for which the network guarantees a certain basic throughput rate, and allows additional traffic at a reduced charge when there is excess capacity. An user subscribing to the service could send as much data as he could, but when he receives a congestion notification, he will throttle the transmission down to the guaranteed rate or risk cell discard by the network.

c) Backward Congestion Notification (BCN): $\mathrm{BCN}$ is used to stop the source from sending more data. This could be a result of back pressure from the upstream network nodes or detection of excess traffic at the $T_{B}$ reference points.

The problem associated with the use of BCN between the network nodes is that the physical path of traffic on the backward direction may not be the same and the congested node may not be on the backward path. Another issue is the vehicle for notification. Piggy-back notification with 
user's data may not always work since there may not be any traffic in the other direction. In this instance, a special traffic management cell for individual connection as indicated by the VPI and VCI in the ATM cell header, or global OA\&M cells would have to be used, which would increase the traffic in the network.

\subsubsection{Media Access Control}

This control is needed when there is more than one B-TE sharing the transmission media within the customer premise. The expectation is that the same ATM protocol would be used for ring, bus, or star configuration. Therefore, the access control mechanism must be under the control of the ATM header, namely the Generic Flow Control field (GFC). This is why the standards groups, T1 and CCITT, use the term "GFC" for media access control.

When a B-TE accepts a call from a remote end, it must be able to handle all traffic from that source. The GFC operation must be designed to control the traffic towards the network and accept all traffic in the other direction.

One of the parameters for controlling the traffic (see next section) is the cell arrival rate, in particular the peak rate. Each B-TE must regulate this rate so that it remains within the subscribed or negotiated range. The GFC function, besides coordinating access to the medium, must perform a traffic smoothing function as well. It is very possible that, due to buffering, the traffic pattern after GFC operation may be more bursty than the traffic generated by an application.

\subsubsection{Traffic Control}

This section discusses other aspects of traffic management: a) connection admission control, b) traffic policing, and c) traffic shaping. Some of these items are implementation issues which are not subject to standardization. Nevertheless, they are important areas for research.

a) Connection Admission Control (CAC): One of the most effective ways of preventing congestion is by controlling the number of connections in the network. The basic idea is that the network may block a call request if it does not have the resources to satisfy the Quality of Service requested. A network may also "shed" some connections when there is a serious congestion or contention for resources. A CAC policy that is too conservative may lead to under utilization of resources and too many blocked calls; on the other hand, a liberal policy may lead to an overload condition and contribute to poor network performance. The issues to be considered in CAC policies include:

- Resource provisioning and tariff management -- this includes proper network capacity planning and resource deployment to meet the customers' demand, and determination of tariffs to meet or change the demand. 
- Network management control -- mechanism to keep track of resources and monitor their usage in real time.

- Call control signalling -- signalling protocols for call setup and disconnect and for describing the service required.

- Definition of traffic descriptors and Quality of Service (QoS) -- a traffic descriptor defines the characteristics of traffic a user may generate. Typical parameters for the descriptor are peak or mean cell rate, duration of peak, cell delay (jitter) variation, cell loss priority, connection priority, and burstiness. They may be defined in terms of traffic at the $T_{B}$ reference point, at the layer boundary between ATM and Physical layers, or between AAL and ATM layers.

- Routing and Load Balancing -- when adding a new connection, the network may preassign a route that has least probability of congestion, or a route that evenly distribute the network load.

b) Traffic Policing Function: The traffic policing function is called Usage/Network Parameter Control (UPC/NPC) in the draft CCITT recommendation I.371. It consists of a set of actions taken by the network to monitor and control traffic in terms of negotiated traffic descriptor for a given connection. This function is performed at the entry to networks as shown by the shaded boxes in Figure 1 for both network A and B. Two major criteria exist for the selection of policing algorithms: (1) being able to respond rapidly to parameter violations, and (2) being able to provide accurate traffic monitoring in real time.

In response to parameter violation, the UPC/NPC function may: (1) discard cells, (2) tag cells for discard (that is, change the CLP bit in the ATM cell header to "eligible for discard" status), (3) initiate Forward Congestion Notification and/or Backward Congestion Notification, or (4) release the connection. The UPC/NPC may also participate in the traffic shaping function, described in the following section, to smooth out the traffic.

Policing could be performed on an individual connection, based on Virtual Channel, or a group of connections on a Virtual Path.

c) Traffic Shaping Control: To reduce burstiness of traffic, traffic shaping control may be applied at the application, AAL, ATM, or UPC/NPC level.

Traffic shaping may be performed as part of the UPC/NPC function to (1) compensate for cell delay variation (CVD) due to effects of multiplexing, physical layer overhead and insertions of OA\&M cells, and (2) reshape the traffic at the entrance of the network for better bandwidth management as well as support of the CVD requirement at the output side.

Traffic shaping is also desirable at the application level from which the traffic is generated. As an example, a video source may compress and packetize the video signals and transmit them on 
a Variable Bit Rate connection. Even though the bit stream for each frame must be delivered to the destination video decoder within a limited delay period, individual packets may not necessarily be delivered as soon as they are generated. Based on the characteristics of the compression algorithm and the knowledge of the frame contents, the B-TE can delay transmission of packets to smooth out the traffic, thus, in effect, reduce the peak cell rate.

\subsection{Fairness of Traffic Management Mechanisms}

One of the major characteristics of an ATM network is the sharing of resources among many classes of users with service requirements ranging from $\mathrm{CBR}$, with strong timing requirement, to VBR, with no timing requirement. Fairness is another important issue in the design of traffic management mechanisms.

Traditionally, in a LAN environment "fairness" means that every station has the same opportunity for access to the shared resources (e.g. transmission media); that is, when the network is fully loaded, all stations should have the same access opportunity and throughput rate. Obviously, this definition is not appropriate in the B-ISDN's multi-media, multi-service environment. Fairness is meaningful only within the same priority class; criteria for fairness may not be the same for a different priority class. For example, VBR for video and VBR for data may have to be considered separately, because dropped cells for the former do not require retransmission, while those for the later would eventually be retransmitted.

\section{CURRENT TRAFFIC MANAGEMENT PROPOSALS FOR B-ISDN STANDARDS}

Standards activities on B-ISDN traffic management concentrate on the ATM layer protocol, because it is the protocol that transports cells across the network. The AAL layer protocol is for communications between two B-TEs, but so far there is no provision in the AAL for end-to-end traffic control. Work on call control signalling and OA\&M cells have started, but with no significant output yet. No work has been done on flow control due to the general feeling that the traditional windowing procedures would not be effective in the B-ISDN environment.

The following sections describe briefly current work by T1 and CCITT in congestion control, general traffic control with traffic descriptors, traffic policing, and media access control (i.e.,GFC). Since T1 prepares input to CCITT, the CCITT Recommendations apply to T1 as well. A more detailed description of current work is presented in other NIST reports to be published [5].

\subsection{Congestion Control}

The ATM protocol as defined by the CCITT has reserved two fields in the ATM header for congestion control [S4,S7]: Cell Loss Priority (CLP) bit and explicit Forward Congestion 
Notification (FCN) bit. Network nodes could discard a cell if its CLP bit is set and notify the destination of the congestion by setting the FCN flag in the Payload Type field of the cell header.

Backward congestion control $(\mathrm{BCN})$ is not defined for reasons stated in Section 4.2.2.

\subsection{Traffic Control}

Draft CCITT Recommendation I.371 [S4] contains definitions of traffic parameters and a description of the connection admission control (CAC) mechanism. Currently, peak cell rate is the only defined traffic parameter.

ATM defines the concept of virtual channel and virtual path that is useful for routing control and traffic policing function.

A number of UPC/NPC algorithms have been proposed and listed in I.371 but none has been selected as the preferred traffic policing method. The following is a brief description of some of the algorithms proposed.

- Leaky Bucket: The Leaky Bucket algorithm accepts cells from the source and controls the average rate of flow in a session with a certain allowable burst rate. The algorithm consists of a bucket capacity or threshold $c$, and a leak rate $r$. The algorithm maintains a counter $a$ which is incremented as cells arrive, and is decremented periodically by an amount equal to $r$. Whenever the rate of flow coming into the bucket exceeds the leak rate for a sufficiently long period of time, that is when $a>c$, the bucket will overflow and the overflowing cells will be marked as a violation of the algorithm. The algorithm uses peak cell rate and Cell Delay Variation (CVD) to determine parameters $c, r$, and the time interval between two decrements. Other refinements to the algorithm, such as Dual Leaky Bucket, have been proposed.

- Jumping Window: In this scheme, a traffic counter is incremented when a cell arrives within the time window (fixed time interval) and resets to zero at the end of the window. When the counter exceeds a predetermined threshold, the cell is either discarded or tagged for discard.

- Triggered Jumping Window: In this scheme, the time windows are triggered by the first arriving cell. This eliminates the ambiguity of source synchronization for the time window and windows are not necessarily consecutive.

- Moving Window: In this scheme, the maximum number of cell arrivals within a window (W time slots) is limited like the jumping window scheme. The adjacent windows, however, overlap by $\mathrm{W}-1$ slots. 
- Spacer-Controller: Incoming cells are actively spaced out by a Spacer-Controller based on the negotiated peak rate. Cells that arrive too closely are either buffered or discarded depending on the upstream CDV requirement.

- Flag Algorithm: In this algorithm, the network or B-TE issues special markers periodically, called UPC flags, and the B-TE can send cells up to the specified number of cells within the flag interval. The UPC control simply counts the number of cells transmitted between adjacent UPC flags to police the traffic rate on a given UNI.

\subsection{Media Access Control (Generic Flow Control)}

There were several GFC protocols submitted to T1 and CCITT. Since CCITT Study Group XVIII requires that the final algorithm accepted must work equally well under three possible configurations (star, ring, and bus), each of the proposals was adapted from the configuration for which the protocol was originally designed. Proposals from AT\&T-USA [W7,W14,W16], Bellcore-USA [W2,W3,W4], and QPSX-Australia [W1,W12,W15] are based on the IEEE 802.6 Distributed Queue Dual Bus (DQDB) [S9] protocol; those from British Telecom-UK [W8,W9,W10,W11,W13], NTT-Japan [W17], and Swiss PTT [W19] are ringbased protocols. Recently Australia had made a new proposal to make GFC independent of media access control by using a stop-and-go mechanism [W21]. The following is a brief description of some of these proposals.

- AT\&T GFC: Since this is based on the DQDB MAC protocol, each B-TE on the bus must place a reservation in the terminal-to-network direction for each cell it wants to transmit as in DQDB, and place the cell in an empty slot when reservations from the downstream terminals have been satisfied. Unlike DQDB, cells have different transmission priorities and are placed in different priority queues depending on the type of services. There are three priority queues, in decreasing order of priority: CBR with low CDV traffic, CBR with high CDV traffic, and VBR traffic. Cell priorities are classified according to a set of parameters: CBR rate, guaranteed VBR rate, burst parameter, and balancing parameter. The balancing parameter is needed to compensate for the positional bias that is inherent in DQDB type protocol. No request for a given priority class will be accepted if there are high priority reservations outstanding.

- British Telecomm MSFC: This proposal, called Multi-Service Flow Control (MSFC), is based on the Owell Ring protocol. Initially all terminals operate without any control in an unrestricted access mode. When a congestion condition is detected, one of the terminals would become a "master" and cause all terminals to switch to restricted access mode (RAM). During the RAM mode, the master issues a sequence of 'reset' cells that control a counter in each terminal. The value of this counter is the number of cells that can be transmitted between 'resets'. Altering the reset rate slows down all terminals as 
necessary, without the master having any knowledge of active terminals or capacity assignment.

\section{SUMMARY}

Clearly, the robustness of B-ISDN/ATM networks depends on effective traffic management. Many factors make the task difficult: the wide range of possible services with varying traffic patterns, high transmission speed, potential high degree of sharing of network resources, and others. No single mechanism can accomplish all of these tasks. A system of network and end user controls is required. End users include the customer premises equipment and related application process. The end user control must coordinate with and compliment the network control. This control must work in conjunction with the customers' service contract, applications' traffic profile, and service providers' tariff (pricing) structure.

Past standards work has established a framework for traffic management. However, much more work in this area needs to be done, however, before implementation of a public switched network. Even though there are emerging standards for ATM switches for private networks, the traffic management mechanisms employed are primitive and proprietary.

\section{REFERENCE}

\section{Articles and Papers}

[1] K. Bala, "Congestion Control for High Speed Packet Switched Networks," IEEE INFOCOM'90, pp 520-526.

[2] K. Bala and K. Sohraby, "Congestion Control for High Speed Packet Switched Networks," IEEE GLOBECOM'90, pp 520-526, December 1990.

[3] A. Berger, A. E. Eckberg, et al., "Performance Characterizations of Traffic Monitoring, and Associated Control, Mechanisms for Broadband Packet Networks," IEEE GLOBECOM'90, pp 350-354.

[4] J. Le Boudec, "The Asynchronous Transfer Mode: a tutorial," Computer Networks and ISDN Systems 24 (1992), pp 279-309, May 1992.

[4] Z. L. Budrikis, "A Generic Flow Control Protocol for B-ISDN," IEEE INFOCOM'92, pp 895-904. 
[5] Y. Chang and D. Su, Study of Generic Flow Control in B-ISDN, NIST Internal Report, October 1992.

[6] H. Chao, "Architecture Design for Regulating and Scheduling User's Traffic in ATM Networks," SIGCOMM'92, Vol. 22, No. 4, pp 77-86, 1992.

[7] R. Despres, "Global Speed WAN Architecture for the 90's," Computer Networks and ISDN Systems, 23 (1991), pp 125-128.

[8] R. Dighe, "Congestion Avoidance Strategies in Broadband Networks," IEEE INFOCOM" 91, pp 295-303.

[9] A. E. Eckberg, "Meeting the Challenge: Congestion and Flow Control Strategies for Broadband Information Transport," IEEE GLOBECOM'89, pp 1769-1773.

[10] G. Gallassi, "Bandwidth Assignment in Prioritized ATM Networks," IEEE GLOBECOM'90, pp 852-856, November 1990.

[11] G. Gallassi, "ATM: Bandwidth Assignment and Bandwidth Enforcement Policies," IEEE GLOBECOM'89, pp 1788-1793, November 1989.

[12] A. Gersht, "A Congestion Control Framework for ATM Networks," IEEE GLOBECOM'89, pp 701-710.

[13] O. Gihr, "A Layered Description of ATM Cell Traffic Streams and Correlation Analysis," IEEE INFOCOM'91, pp 137-144.

[14] D. Hong and T. Suda, "Congestion Control and Prevention in ATM Networks," IEEE Network, pp 10-16, July 1991.

[15] K. Kawashima, "Teletraffic Issues in ATM Networks," Computer Networks and ISDN Systems, 20 (1990), pp 369-375.

[16] S. Kowtha and D. Vaman, Realization of a Multimedia Traffic Generator based on $(u, a)$ Buffer Occupancy Analysis, Internal Memo, Stevens Institute of Technology, April 1992.

[17] H. Kroner, "Comparative Performance Study of Space Priority Mechanisms for ATM Networks," IEEE INFOCOM'90, pp 1136-1143, 1990.

[18] F. Kuo, Protocols and Techniques for Data Communications Networks, pp 122-169, Prentice Hall, 1981. 
[19] W. Leland, "Window-Based Congestion Management in Broadband ATM Networks," IEEE GLOBECOM'89, pp 1794-1800, November 1989.

[20] B. Makrucki, "Forward Congestion Notification in ATM Networks," 3rd Annual Workshop on Very High Speed Networks, pp 137-148, March 9, 1992.

[21] P. Mishra and H. Kanakia, "A Hop by Hop Rate-Based Congestion Control Scheme," Proceedings of SIGCOMM'92, August 17, 1992.

[22] S. Morgan, "Queueing Disciplines and Passive Congestion Control in Byte-Stream Networks," IEEE INFOCOM'89, pp 711-720.

[23] G. Ramamurthy and R. Dighe, "A Multidimensional Framework for Congestion Control in B-ISDN," IEEE Journal on Selected Areas in Communications, Vol. 9, No. 9, pp 1440-1451, December 1991.

[24] E. P. Rathgeb, "Modeling and Performance Comparison of Policing Mechanisms for ATM Networks," IEEE Journal on Selected Areas in Communications, Vol. 9, No. 3, pp 325-334, April 1990.

[25] M. Schroeder, et al., A High-Speed, Self-Configuring LAN Using Point-to-Point Links, Stanford Research Center, Digital Equipment Corporation, April 30, 1990.

[26] W. Stallings, Data and Computer Communications, MacMillan Publishing, 3rd Edition, 1991.

[27] H. Suzuki and S. Sato, "A Burst Traffic Control Strategies for ATM Networks," IEEE GLOBECOM'90, pp 874-878.

[28] A. Tanenbaum, Computer Networks, Prentice Hall, 2nd Edition, 1988.

[29] A. Vasilakos, "Variable Window Flow Control and Ergodic Discretized Learning Algorithms for Adaptive Routing in Data Networks," Computer Networks and ISDN Systems, 22 (1991), pp 235-248.

[30] G. M. Woodruff, "Multimedia Traffic Management Principles for Guaranteed ATM Network Performance," IEEE Journal on Selected Areas in Communications, Vol. 8, No. 3, pp 400-437, April 1990.

[31] L. Zhang, "A New Traffic Control Algorithm for Packet Switching Networks," Proceedings of SIGCOMM'90, pp 19-29, 1990. 
[32] L. Zhang, "A Comparison of Traffic Control Algorithm for High-Speed Networks," 2nd Annual Workshop on Very High Speed Networks, 1991.

[33] J. Roberts, COST 224 Project Report: Performance Evaluation and Design of Multiservice Networks, Directorate-General, Telecommunications, Information Industries and Innovation, Commission of the European Communities, October 1991.

\{34] R. Falconer, J. Adams, "Orwell: a protocol for an integrated services local network," British Telecom Technol Journal, Vol. 3, No. 4, October 1985.

ANSI Standards and CCITT Recommendations (Approved and Draft)

[S1] CCITT Recommendation I.150, B-ISDN ATM Functional Characteristics, June 1992.

[S2] CCITT B-ISDN Recommendation I.211, B-ISDN Service Aspects, June 1992.

[S3] CCITT Recommendation I.361, B-ISDN ATM Layer Specification, June 1992.

[S4] CCITT Recommendation I.371, Traffic Control and Congestion Control in B-ISDN, June 1992.

[S5] CCITT Recommendation I.413, B-ISDN User-Network Interface, June 1992.

[S6] T1S1/91-634R1, ANSI T1.INF-199X, Broadband ISDN User-Network Interfaces: Rates and Formats Specifications, May, 1992.

[S7] T1S1/92-457, ANSI T1.ATM-199X DRAFT, B-ISDN ATM Layer Functionality and Specification, August 29, 1992.

[S8] ANSI T1.105-1991, Digital Hierarchy - Optical Interface Rates and Formats Specifications (SONET), July 2, 1991.

[S9] IEEE Std 802.6-1990, Distributed Queue Dual Bus (DQDB) Subnetwork of a Metropolitan Area Network (MAN), July 3, 1991.

\section{T1S1 Working Documents}

[W1] T1S1.5/90-080, QPSX, Draft Text for the QPSX Proposed Structure and Semantics of GFC, March 1991.

[W2] T1S1.5/90-185, Bellcore, Proposal For a GFC Protocol, August 27, 1990. 
[W3] T1S1.5/90-188, Bellcore, SDL Diagrams of the BDFC Protocol, August 27, 1990.

[W4] T1S1.5/90-187, Bellcore, Initial Performance Study of the BDFC Protocol, August 27, 1990.

[W5] T1S1.5/90-263, Bellcore, Traffic Shaping, October 29, 1990.

[W6] T1S1.5/90-264 \& 265, Bellcore, Performance of a Traffic Shaper, Simulation Results, October 29, 1990.

[W7] T1S1.5/90-274, AT\&T, Use of the Generic Flow Control Field, October 29, 1990.

[W8] T1S1.5/90-160, British Telcom, Multiservice GFC Protocol with Improved Reset Mechanism, June 25, 1990.

[W9] T1S1.5/91-151, British Telcom, General Description of the Multi-Service Flow Control Protocol, May 6, 1991.

[W10]T1S1.5/91-152, British Telecom, GFC-V2 SDL Diagrams for the MSFC Protocol, May 6, 1991.

[W11]T1S1.5/91-155, British Telcom, Simplified Terminals Incorporating GFC based on MSFC Protocol, May 6, 1991.

[W12]T1S1.5/91-220, QPSX, Unassigned Bandwidth Utilization Under QPSX Proposed GFC Protocol, May 6, 1991.

[W13]T1S1.5/91-370, British Telcom, BT-NTT Proposed GFC Protocol, 1, November 4, 1991.

[W14]T1S1.5/91-390, AT\&T, GFC Proposal and Simulation Results, November 4, 1991.

[W15]T1S1.5/91-393, QPSX, Australia Backed Proposal of GFC Protocol, November 4, 1991.

[W16]T1S1.5/91-421, AT\&T, Use of the Generic Flow Control Field, November 4, 1991.

[W17]T1S1.5/92-007, British Telcom, BT-NTT Combined Proposal for GFC, October 9, 1991.

[W18]T1S1.5/92-009, "Working Document of Traffic Management/Congestion Control," January 20, 1992.

[W19] CCITT D.1434, NTT, "Proposal for GFC Protocol," June 10, 1991. 
[W20] CCITT D.1441, Swiss_PTT, "A Candidate GFC Protocol," June 10, 1991.

[W21] CCITT D.xxxx, Australia-QPXX, "A Controlled Point-to-Point Generic Flow Control Protocol," June 1992. 


